\title{
Phenomenological predictions of the properties of the $B_{c}$ system
}

\author{
Lewis P. Fulcher \\ Department of Physics and Astronomy, Bowling Green State University \\ Bowling Green, Ohio 43403 \\ email: fulcher@newton.bgsu.edu \\ and \\ Institut für Theoretische Physik der Justus Liebig Universität \\ D-35392 Gießen, Germany
}

\begin{abstract}
We present a comprehensive calculation of the energies, splittings and electromagnetic decays of the low-lying levels of the bottom charmed meson system. In order to incorporate running coupling constant effects, we choose Richardson's potential for the central potential and take the spin-dependent potentials from the radiative one-loop calculation of Pantaleone, Tye and Ng. The effects of a nonperturbative spin-orbit potential are also included. Our parameters are determined from the low-lying levels of charmonium (avg. dev. of $19.9 \mathrm{MeV}$ ) and of the upsilon system (avg. dev. of $4.3 \mathrm{MeV}$ ). We carry out a detailed comparison with the calculations of Eichten and Quigg and the lattice calculations of the NRQCD collaboration. Our predicted result for the ground state energy is $6286_{-6}^{+15} \mathrm{MeV}$. Our results are generally in agreement with the earlier calculations. However, we find the two lowest $1^{+}$states to be very close to the $\mathrm{j}$-j limit, in contrast to some of the earlier calculations. The implications of this finding for the photon spectra of the $1 \mathrm{P}$ and $2 \mathrm{~S}$ states are discussed in detail. Some strategies for the observation of these states are discussed, and a table of their cascades to the ground state is presented. Our calculated value for the ground state lifetime is $0.38 \pm 0.03 \mathrm{ps}$, in good agreement with the recent CDF measurement.
\end{abstract}

12.39.Pn,14.40.Gx,14.40.Lb,14.40.Nd

\section{INTRODUCTION}

The CDF collaboration 11] has reported the discovery of the $B_{c}$ system in $1.8 \mathrm{TeV} p-\bar{p}$ collisions at Fermilab. They have observed about 20 decays in the $J / \psi$-lepton channel, which are interpreted as decays of the ground state. For the mass of the ground state, the CDF collaboration quotes $M_{B_{c}}=6.40 \pm 0.39 \pm 0.13 \mathrm{GeV}$. Their value for the lifetime is $\tau_{B_{c}}=0.46_{-0.16}^{+0.18} \pm 0.03 \mathrm{ps}$. This state should be one of a number of states lying below the threshold for emission of $\mathrm{B}$ and $\mathrm{D}$ mesons. Because these states can not decay by gluon annihilation, they should be very stable in comparison with their counterparts in charmonium and the upsilon system.

The purpose of this report is to give a detailed account of the energies, splittings and electromagnetic decay rates for the $B_{c}$ states below the continuum threshold and to propose strategies for detecting some of these states. We will use a potential model that includes running coupling constant effects in both the central potential and the spin-dependent potentials to give a simultaneous account of the properties of charmonium, the upsilon system and the $B_{c}$ system. We choose Richardson's potential [2] to represent the central potential and insist upon strict flavor-independence of its parameters. Since one would expect the average values of the momentum transfer in the various quark-antiquark states to be different, some variation in the values of the strong coupling constant and the renormalization scale in the spin-dependent potentials should be expected. In order to minimize the role of flavor-dependence, we use the same values for the coupling constant and the renormalization scale for each of the levels in a given system and require that these values be consistent with a universal QCD scale. Since one can calculate the central potential and the spin-dependent potentials from first principles on the lattice [3], it should be possible at some point in future calculations to connect subsets of these potential parameters in more fundamental ways. Such a program would be an important step towards finding a more rigorous way to formulate a potential model calculation.

In $1991 \mathrm{Kwong}$ and Rosner [1] predicted the masses of the lowest vector and pseudoscalar states of the $B_{s}$ and the $B_{c}$ systems using an empirical mass formula and a logarithmic potential. Eichten and Quigg [5] gave a more comprehensive account of the energies and decays of the of the $B_{c}$ system that was based on the QCD-motivated potential of Buchmüller and Tye [6]. Gershtein et al. [7] also published a detailed account of the energies and decays of the $B_{c}$ system and established contact with QCD sum-rule calculations. Both of these latter calculations included running coupling constant effects in the central potential, but used spin-dependent potentials that were restricted to the tree level.

One of the most important goals of the present calculation is to extend the treatment of the spin-dependent potentials to the full radiative one-loop level and thus include effects of the running coupling constant in these potentials. In 
a previous calculation [8] we have shown that such effects offer a substantial improvement in the calculation of the spectra of charmonium and the upsilon system. In particular, it becomes possible to offer a good account of both the fine structure splittings and the hyperfine structure. Our previous calculation used the renormalization scheme developed by Gupta and Radford [9, 10. However for this calculation, we have chosen the modified-minimal subtraction scheme used by Pantaleone, Tye and Ng (PTN) 111 to extend their perturbative QCD calculation to the one-loop level. We supplement the PTN formalism with a long-range spin-orbit potential to be consistent with the Gromes consistency condition [12] and recent lattice calculations [3]. The flavor dependence allowed the string constant in the 1991 calculation is not permitted in the present calculation. Thus this calculation should also be viewed as part of the effort to formulate potential model calculations under a more restrictive set of assumptions. We note in passing a number of additional important calculations of the properties of the $B_{c}$ system [13 17 .

In Sect. III we present preliminary calculations of the lowest vector and pseudoscalar states of the $B_{c}$ system with several potential models. These exercises are similar to some carried out by Eichten and Quigg [5] and represent one way of estimating the uncertaintities in the predicted energies of the new system. In Sect. III we introduce Richardson's potential and the expressions necessary to include the one-loop corrections in the spin-dependent potentials and discuss the determination of our parameters from charmonium and the upsilon system. The formalism required for the mixing of the $\mathrm{P}$ states in the $B_{c}$ system is presented in Sect. IV. The last section contains our results for the energies and decays of the low-lying $B_{c}$ states and a discussion of their implications for the development of strategies to detect these states.

\section{SOME POTENTIAL MODELS}

The three systems that we consider in this paper, upsilon, charmonium and $B_{c}$, are often considered as nonrelativistic systems, and thus our treatment is based upon the Schrödinger equation with a Hamiltonian of the form,

$$
H=p^{2} / 2 \mu+V(r)+V_{\mathrm{SD}}
$$

where $\mu=m_{1} m_{2} /\left(m_{1}+m_{2}\right)$ is the reduced mass. No spin-independent relativistic corrections are included. For the purpose of making some preliminary estimates of the energies of the lowest two states of the $B_{c}$ system, it is necessary to consider only the spin-spin part of the spin-dependent potential since these are $\mathrm{S}$ states. Thus,

$$
V_{S D} \rightarrow V_{S S}=\frac{32 \pi \alpha_{S}}{9 m_{1} m_{2}} \delta^{3}(\mathbf{r}) \mathbf{S}_{1} \cdot \mathbf{S}_{2}
$$

Our solutions to the Schrödinger equation are generated numerically with the given form for the central potential [18] and the effects of the spin-dependent parts are added as a perturbation improvement. Thus, the 1S-state hyperfine splitting is given by

$$
\Delta E_{\mathrm{hfs}}=\frac{8 \alpha_{S}}{9 m_{1} m_{2}}\left|R_{1 S}(0)\right|^{2}
$$

We address the question of the validity of first-order perturbation theory for the contact potential of Eq. (2) in an Appendix.

All of the potential parameters in this section are strictly flavor-independent. This includes any additive constants. The central potential parameters and the constituent mass parameters are fit to the low-lying energy levels of charmonium and the upsilon system. The strong coupling constant $\alpha_{S}$ is fit to the observed charmonium hyperfine splitting of $117 \mathrm{MeV}$. In Table I below we present results with 5 different central potentials. These include the Cornell potential [19]

$$
V(r)=A r-\kappa / r+C
$$

where $A=0.1756 \mathrm{GeV}^{2}, \kappa=0.52$ and $C=-0.8578 \mathrm{GeV}$; Martín's power-law potential [20],

$$
V(r)=-8.093+6.898(r \times 1 \mathrm{GeV})^{0.1}
$$

(units of the potential are $\mathrm{GeV}$ ), and the logarithmic potential [21],

$$
V(r)=-0.6631+0.733 \ln (r \times 1 \mathrm{GeV}),
$$

(potential units are also GeV). Each of these forms was used by Eichten and Quigg. However, our values for the potential parameters are slightly different. We also have done calculations with the potential of Song and Lin [22], 


$$
V(r)=A r^{1 / 2}-B r^{-1 / 2},
$$

where $A=0.511 \mathrm{GeV}^{3 / 2}$ and $B=0.923 \mathrm{GeV}^{1 / 2}$, and the Turin 23] potential,

$$
V(r)=-a r^{-3 / 4}+b r^{3 / 4}+C,
$$

where $a=0.620 \mathrm{GeV}^{1 / 4}, b=0.304 \mathrm{GeV}^{7 / 4}$ and $C=-0.823 \mathrm{GeV}$.

Our results in the first 3 columns of Table f 1 are very similar to those presented in Table I of Eichten and Quigg [5]. The results obtained with the Song-Lin and the Turin potentials in all cases fall between the extremes defined by the first 3 columns. Averaging over all 5 cases presented in Table I yields

$$
M_{B_{c}}=6251_{-5}^{+15} \mathrm{MeV}, \quad M_{B_{c}^{*}}=6328_{-8}^{+9} \mathrm{MeV},
$$

as the predicted energies of the two lowest states. If we treat Eichten and Quigg's results in the same way, then we get $6258_{-10}^{+8} \mathrm{MeV}$ for $B_{c}$ and $6333_{-14}^{+10} \mathrm{MeV}$ for $B_{c}^{*}$, in good agreement with our preliminary analysis. All of these results fall comfortably within the ranges found by Kwong and Rosner 顿, that is,

$$
\begin{aligned}
& 6194 \mathrm{MeV} \leq M_{B_{c}} \leq 6292 \mathrm{MeV}, \\
& 6284 \mathrm{MeV} \leq M_{B_{c}^{*}} \leq 6357 \mathrm{MeV} .
\end{aligned}
$$

\section{RICHARDSON'S POTENTIAL AND THE SPIN-DEPENDENT POTENTIALS}

The starting point for the derivation [24] of Richardson's potential is the one-loop expression for the running coupling constant [25], namely,

$$
\alpha_{S}\left(\left|\mathbf{q}^{2}\right|\right)=\frac{12 \pi}{\left(33-2 n_{f}\right) \ln \left(\left|\mathbf{q}^{2}\right| / \Lambda^{2}\right)},
$$

where $n_{f}$ denotes the number of quark degrees of freedom accessible to the propagating gluon [26] and $\Lambda$ is the QCD scale. Richardson [2] realized that one could tame the infrared singularity of Eq. (11) and produce a linear confining potential by making the replacement,

$$
\left|\mathbf{q}^{2}\right| / \Lambda^{2} \rightarrow\left|\mathbf{q}^{2}\right| / \Lambda^{2}+1,
$$

in the argument of the logarithm. Our 1991 calculation [8] was based on a modification of Richardson's potential suggested by Moxhay and Rosner [27, that is, the string constant is treated as a free parameter instead of being connected to the scale parameter $\Lambda$. Then the potential takes the form,

$$
V(r)=A r-\frac{8 \pi f(\Lambda r)}{\left(33-2 n_{f}\right) r},
$$

where

$$
f(t)=\frac{4}{\pi} \int_{0}^{\infty} \frac{\sin t x}{x}\left[\frac{1}{\ln \left(1+x^{2}\right)}-\frac{1}{x^{2}}\right] d x .
$$

To specify the spin-dependent potentials, we use a generalization of the Eichten-Feinberg fromalism [13] derived by PTN [11]. Our notation is closely akin to that of Eichten and Quigg [5]. Thus,

$$
V_{S D}=\frac{\mathbf{L} \cdot \mathbf{S}_{1}}{2 m_{1}^{2}} T_{a}+\frac{\mathbf{L} \cdot \mathbf{S}_{2}}{2 m_{2}^{2}} T_{a}^{\prime}+\frac{\mathbf{L} \cdot\left(\mathbf{S}_{1}+\mathbf{S}_{2}\right)}{m_{1} m_{2}} T_{b}+\frac{\mathbf{L} \cdot\left(\mathbf{S}_{1}-\mathbf{S}_{2}\right)}{m_{1} m_{2}} T_{b}^{\prime}+\frac{S_{12}}{m_{1} m_{2}} T_{c}+\frac{\mathbf{S}_{1} \cdot \mathbf{S}_{2}}{m_{1} m_{2}} T_{d},
$$

where $S_{12}$ denotes the tensor operator, that is, $4\left[3 \mathbf{S}_{1} \cdot \hat{\mathbf{r}} \mathbf{S}_{2} \cdot \hat{\mathbf{r}}-\mathbf{S}_{1} \cdot \mathbf{S}_{2}\right]$ and $m_{1}$ denotes the mass of the lighter (charmed) quark, if the two constituent masses are not equal. The quantities T are connected with the PTN potentials as follows:

$$
T_{a}=\frac{1}{r} \frac{d}{d r}(E-A r)+\frac{2}{r} \frac{d V_{1}}{d r}+2 V_{5}, \quad T_{a}^{\prime}=T_{a}-4 V_{5},
$$




$$
T_{b}=\frac{1}{r} \frac{d V_{2}}{d r}, \quad T_{b}^{\prime}=V_{5}, \quad T_{c}=V_{3} / 12, \quad T_{d}=V_{4} / 3,
$$

where we have supplemented the PTN potentials with a long range spin-orbit contribution to include nonperturbative effects. Such a contribution is clearly indicated by the lattice calculations of Bali, Schilling and Wachter [3] and is required to satisfy the consistency condition,

$$
\frac{d}{d r}\left[E(r)+V_{1}(r)-V_{2}(r)\right]=0
$$

derived by Gromes 12] from the requirements of Lorentz covariance.

The PTN potentials are expressed in terms of the strong coupling constant $\alpha_{\bar{S}}$ and the renormalization scale $\mu$. The function $\mathrm{E}(\mathrm{r})$ includes the leading correction to the central potential,

$$
E(r)=-\frac{4 \alpha_{\bar{S}}}{3 r}\left[1+\frac{\alpha_{\bar{S}}}{6 \pi}\left[\left(33-2 n_{f}\right)\left(\ln \mu r+\gamma_{E}\right)+\frac{31}{2}-\frac{5 n_{f}}{3}\right]\right],
$$

where $\gamma_{E}=0.5772 \cdots$, is Euler's constant. This function is of course a part of the contribution to the short-distance behavior of the running coupling constant in Eq. (11), although its contribution to Richardson's potential is not made explicit. The first two spin-orbit potentials are given by

$$
\begin{gathered}
V_{1}(r)=-\frac{\alpha_{\bar{S}}^{2}}{2 \pi r}\left[\frac{16}{9}-4\left(\ln \sqrt{m_{1} m_{2}} r+\gamma_{E}\right)\right] \\
V_{2}(r)=-\frac{4 \alpha_{\bar{S}}}{3 r}\left[1+\frac{\alpha_{\bar{S}}}{6 \pi}\left[\left(33-2 n_{f}\right)\left(\ln \mu r+\gamma_{e}\right)+\frac{39}{2}-\frac{5 n_{f}}{3}-9\left(\ln \sqrt{m_{1} m_{2}} r+\gamma_{E}\right)\right]\right] .
\end{gathered}
$$

The tensor potential takes the form,

$$
V_{3}(r)=\frac{4 \alpha_{\bar{S}}}{r^{3}}\left[1+\frac{\alpha_{\bar{S}}}{6 \pi}\left[\left(33-2 n_{f}\right)\left(\ln \mu r+\gamma_{E}-\frac{4}{3}\right)+\frac{65}{2}-\frac{5 n_{f}}{3}-18\left(\ln \sqrt{m_{1} m_{2}} r+\gamma_{E}-\frac{4}{3}\right)\right]\right] .
$$

The lengthy expression for the spin-spin potential,

$$
\begin{aligned}
V_{4}(r)= & \frac{32 \pi \alpha_{\bar{S}}}{3}\left\{1+\frac{\alpha_{S}}{6 \pi}\left[\frac{11}{2}-\frac{5 n_{f}}{3}-\frac{3}{4}\left(\frac{9 m_{1}^{2}+9 m_{2}^{2}-14 m_{1} m_{2}}{m_{1}^{2}-m_{2}^{2}}\right) \ln \left(m_{2} / m_{1}\right)\right]\right\} \delta^{3}(\mathbf{r}) \\
& -\frac{4 \alpha_{\bar{S}}^{2}}{9 \pi}\left[\left(33-2 n_{f}\right) \nabla^{2}\left(\frac{\ln \mu r+\gamma_{E}}{r}\right)-\frac{63}{2} \nabla^{2}\left(\frac{\ln \sqrt{m_{1} m_{2}} r+\gamma_{E}}{r}\right)\right],
\end{aligned}
$$

reduces to an especially simple form for states with nonzero angular momentum, that is,

$$
V_{4} \rightarrow \frac{\alpha_{\bar{S}}^{2}}{\pi r^{3}}\left[\frac{2}{3}-\frac{8 n_{f}}{9}\right]
$$

since one can take the derivatives present in Eq. (22) in this case, and the delta function there does not contribute. If the two constituent quarks have equal masses, then it is necessary to add a second-order contribution from the annihilation graphs,

$$
\delta V_{4}=8 \alpha_{\bar{S}}^{2}(1-\ln 2) \delta^{3}(\mathbf{r}),
$$

to the expression of Eq. (22). The spin-orbit potential discovered by PTN is given by

$$
V_{5}(r)=\frac{\alpha_{\bar{S}}^{2}}{\pi r^{3}} \ln \left(m_{1} / m_{2}\right) .
$$

The equations describing the PTN potentials use the modified minimal subtraction scheme to define the strong coupling constant $\alpha_{\bar{S}}$. This coupling constant can be related to that defined in a scheme developed by Gupta et al. [9,10], which was used in the 1991 calculation [8], that is,

$$
\alpha_{G R R}\left(\mu^{2}\right)=\alpha_{\bar{S}}\left(\mu^{2}\right)\left[1+\frac{\alpha_{\bar{S}}\left(\mu^{2}\right)}{12 \pi}\left(49-\frac{10 n_{f}}{3}\right)\right]+\cdots,
$$


to the second order in $\alpha_{S}$. It is straightforward to verify that PTN's expressions for the potentials are the same as those of Gupta et al. to the second order in $\alpha_{S}$ by using Eq. (26) to connect the two renormalization schemes. The signs of the terms in Eq. (26) indicate that we should expect smaller coupling constants in the present calculation than in our 1991 calculation.

In determining the matrix elements for the perturbation improvement of the central potential energies, we must exercise some care since the total spin, $\mathbf{S}=\mathbf{S}_{1}+\mathbf{S}_{2}$, is not a good quantum number in the most general case. This is a consequence of the fact that its magnitude does not commute with the spin-dependent potential because, for example,

$$
\left[\mathbf{L} \cdot \mathbf{S}_{1}, S^{2}\right]=2 i \mathbf{L} \cdot\left(\mathbf{S}_{\mathbf{2}} \times \mathbf{S}_{\mathbf{1}}\right)
$$

We will deal with the more complicated case of the mass-mixing matrix in the next section. For the remainder of this section, we will consider only the diagonal matrix elements. This will suffice to determine the requisite expectation values for the case of equal masses, since then the total spin is a good quantum number.

The diagonal matrix elements can all be evaluated in terms of the expectation values $[5]$ of $\langle\mathbf{L} \cdot \mathbf{S}\rangle$ since

$$
\left\langle\mathbf{L} \cdot \mathbf{S}_{1}\right\rangle=\left\langle\mathbf{L} \cdot \mathbf{S}_{2}\right\rangle=\frac{1}{2}\langle\mathbf{L} \cdot \mathbf{S}\rangle
$$

and

$$
\left\langle S_{12}\right\rangle=\frac{4\left\langle L^{2}\right\rangle\left\langle S^{2}\right\rangle-6\langle\mathbf{L} \cdot \mathbf{S}\rangle-12\langle\mathbf{L} \cdot \mathbf{S}\rangle^{2}}{(2 l+3)(2 l-1)}
$$

where $l$ is the orbital angular momentum quantum number, as we have verified [28 30]. Hence, the diagonal matrix elements of the spin-dependent potentials may be expressed

$$
\left\langle V_{S D}\right\rangle=\langle\mathbf{L} \cdot \mathbf{S}\rangle\left[\frac{\left\langle T_{a}\right\rangle}{4 m_{1}^{2}}+\frac{\left\langle T_{a}^{\prime}\right\rangle}{4 m_{2}^{2}}+\frac{\left\langle T_{b}\right\rangle}{m_{1} m_{2}}\right]+\frac{1}{m_{1} m_{2}}\left[\left\langle S_{12}\right\rangle\left\langle T_{c}\right\rangle+\left\langle\mathbf{S}_{1} \cdot \mathbf{S}_{2}\right\rangle\left\langle T_{d}\right\rangle\right]
$$

Our results for the charmonium and upsilon energies obtained with Richardson's potential and the one-loop expressions for the spin-dependent potentials are presented in Tables II and III (listed in column 4 as FUII98), where they are compared with the results of Eichten and Quigg [5]. The flavor-independent potential parameters and constituent masses used to obtain these results are

$$
\begin{aligned}
A & =0.152 \mathrm{GeV}^{2}, \quad \Lambda=0.431 \mathrm{GeV}, \\
m_{b} & =4.889 \mathrm{GeV}, \quad m_{c}=1.476 \mathrm{GeV} .
\end{aligned}
$$

For the upsilon system the value of the coupling constant $\alpha_{\bar{S}}=0.30$, and the value of the renormalization scale $\mu=1.95 \mathrm{GeV}$. For charmonium these values are $\alpha_{\bar{S}}=0.486$ and $\mu=0.80 \mathrm{GeV}$. In both cases the value of the universal QCD scale determined from

$$
\Lambda_{Q C D}=\mu e^{-6 \pi /\left(33-2 n_{f}\right) \alpha_{\bar{S}}},
$$

is $0.190 \mathrm{GeV}$, a value consistent with other determinations [31. Agreement between the calculated results and the measured values [31] in Tables III and III is extremely good, the average deviation being only $4.3 \mathrm{MeV}$ for the upsilon system. Most of the $19.9 \mathrm{MeV}$ deviation in the charmonium case arises from a $25 \mathrm{MeV}$ discrepancy in the center-ofgravity of the $1 \mathrm{P}$ state. Much of this difference could probably be removed by a proper account of the spin-independent relativistic corrections [32].

Our results for the upsilon and charmonium leptonic widths are presented in Table IV, where they are compared with those calculated by Eichten and Quigg [5]. Our decay rates were obtained with the formula [33],

$$
\Gamma_{e e}=\frac{4 \alpha^{2} e_{Q}^{2}}{M^{2}(Q \bar{Q})}|R(0)|^{2}\left[1-\frac{16 \alpha_{S}}{3 \pi}\right]
$$

where $\alpha$ is the fine structure constant, $e_{Q}$ denotes the quark charge, and $\mathrm{M}$ denotes the mass of the quark-antiquark state. Since the average values of the momentum transferred in the annihilation graphs underlying Eq. (33) should be much larger than that associated with the scattering processes of the spin-dependent potentials above, we should expect the appropriate value for $\alpha_{S}$ in Eq. (33) to be smaller than that used above for the fine structure splittings. 
Thus we choose $\alpha_{S}=0.18$, a value obtained from heavy quarkonium decays [8.34]. Our leptonic widths also agree with experiment very well. In order to carry out a fair comparison with the widths of Eichten and Quigg, it is necessary to correct their published results for radiative QCD corrections. Doing this as described above gives the EQ results shown in parentheses and moves their results much closer to the measured values. The differences between the values listed in columns 2 and 3 of Table IV gives a measure of the differences in wave functions calculated with Richardson's potential and the Buchmüller-Tye potential.

In order to have a good basis for comparison, we have carried out a Richardson's potential calculation with tree-level expressions for all the spin-dependent potentials. These may be readily obtained by omitting all terms of $O\left(\alpha_{\bar{S}}^{2}\right)$ in the spin-dependent potentials above, and thus there is no reference to the renormalization scale. Then the expressions for the functions $\mathrm{T}$ are especially simple, that is,

$$
\begin{gathered}
T_{a}=T_{a}^{\prime}=\frac{4 \alpha_{\bar{S}}}{3 r^{3}}-\frac{A}{r} \\
T_{b}=\frac{4 \alpha_{\bar{S}}}{3 r^{3}}, \quad T_{b}^{\prime}=0, \quad T_{c}=\frac{\alpha_{\bar{S}}}{3 r^{3}}, \quad T_{d}=\frac{32 \pi \alpha_{\bar{S}}}{9} \delta^{3}(\mathbf{r}) .
\end{gathered}
$$

The central potential parameters in this calculation are the same as those listed in Eq. (31). The constituent mass for the charmed quark is $1.476 \mathrm{GeV}$, the same as in Eq. (31), and $m_{b}=4.884 \mathrm{GeV}$, slightly smaller. The coupling constant $\alpha_{S}=0.339$. Results of this calculation are also presented in Tables II and III (designated as FUI98 in column 3). For both charmonium and the upsilon system, the average deviation is smaller for the one-loop calculation, but this does not tell the whole story. The fit to the fine structure splittings of charmonium with the one-loop calculation is much better than either of the tree-level calculations presented in Table III. This is our basis for the expectation that a one-loop calculation should give a more accurate rendering of the fine structure and the hyperfine structure of the $B_{c}$ system. Since the central potential parameters are the same, the leptonic widths for the FUI and FUII calculations are the same.

One of the most dramatic differences in the one-loop and tree level results is in the prediction for upsilon hyperfine splittings in Table III. For example, the one-loop prediction for the $1 \mathrm{~S}$ state is $55 \mathrm{MeV}$, which is consistent with earlier predictions [8,10] and the average of the tree-level predictions is $90 \mathrm{MeV}$. It is interesting to note that the one-loop level prediction is much closer to the lattice results $(44-50 \mathrm{MeV})$ of Bali, Schilling and Wachter [3]. Thus, measurement of the energies of the singlet $\mathrm{S}$ energies would serve to clarify the proper input for a good phenomenological calculation of the properties of heavy quark systems. Some experimental group should give these measurements a high priority.

\section{MASS MIXING MATRIX FOR P STATES}

In determining the eigenvalues and eigenfunctions for the $B_{c}$ system, we have a choice of using basis functions from either the L-S coupling scheme or the $\mathrm{j}$-j coupling scheme. We follow the lead of Eichten and Quigg [5] and choose the $\mathrm{j}$-j basis. First we form the total angular momentum of the charmed quark, $\mathbf{J}_{1}=\mathbf{L}+\mathbf{S}_{1}$, and then we form the

total angular momentum of the system, $\mathbf{J}=\mathbf{J}_{1}+\mathbf{S}_{2}$. For P states, the $J=2$ states and the $J=0$ state are the same in either basis, that is,

$$
\psi_{2 m}\left(\frac{3}{2} \frac{1}{2}\right)=\psi_{2 m}(11), \quad \psi_{00}\left(\frac{1}{2} \frac{1}{2}\right)=\psi_{00}(11),
$$

and the determination of the eigenvalues of the spin-dependent potentials requires only the diagonal elements of Eq. (30). On the other hand, the $J=1$ states are a combination,

$$
\psi_{1 m}=a_{1} \psi_{1 m}\left(\frac{3}{2} \frac{1}{2}\right)+a_{2} \psi_{1 m}\left(\frac{1}{2} \frac{1}{2}\right),
$$

and one must diagonalize the mass mixing matrix in order to determine the eigenvalues and eigenfunctions. In the basis described above, we have derived the following forms for the spin-dependent operators of Eq. (15), that is,

$$
\left(\mathbf{L} \cdot \mathbf{S}_{1}\right)=\left(\begin{array}{cc}
\frac{1}{2} & 0 \\
0 & -1
\end{array}\right), \quad\left(\mathbf{L} \cdot \mathbf{S}_{2}\right)=\left(\begin{array}{cc}
-\frac{5}{6} & -\frac{\sqrt{2}}{3} \\
-\frac{\sqrt{2}}{3} & \frac{1}{3}
\end{array}\right)
$$

and 


$$
\left(S_{12}\right)=\left(\begin{array}{cc}
\frac{2}{3} & \frac{2 \sqrt{2}}{3} \\
\frac{2 \sqrt{2}}{3} & \frac{4}{3}
\end{array}\right), \quad\left(\mathbf{S}_{1} \cdot \mathbf{S}_{2}\right)=\left(\begin{array}{cc}
-\frac{5}{12} & \frac{\sqrt{2}}{3} \\
\frac{\sqrt{2}}{3} & -\frac{1}{12}
\end{array}\right) .
$$

To obtain the matrices for the operators $\mathbf{L} \cdot \mathbf{S}$ and $\mathbf{L} \cdot\left(\mathbf{S}_{1}-\mathbf{S}_{2}\right)$, one simply takes the appropriate linear combination of the matrices in Eqs. (37a). By inspection one can verify that our first 3 matrices in Eqs. (37) are consistent with Eqs. (2.21) of Eichten and Quigg [5].

It is straightforward to take the equal-mass, or L-S, limit. Then one can find a unitary transformation that simultaneously diagonalizes the operators, $\mathbf{L} \cdot \mathbf{S}, S_{12}$ and $\mathbf{S}_{1} \cdot \mathbf{S}_{2}$. The eigenvectors are given by

$$
\begin{aligned}
& \psi_{1 m}\left({ }^{3} P_{1}\right)=\sqrt{\frac{1}{3}} \psi_{1 m}\left(\frac{3}{2} \frac{1}{2}\right)+\sqrt{\frac{2}{3}} \psi_{1 m}\left(\frac{1}{2} \frac{1}{2}\right), \\
& \psi_{1 m}\left({ }^{1} P_{1}\right)=\sqrt{\frac{2}{3}} \psi_{1 m}\left(\frac{3}{2} \frac{1}{2}\right)-\sqrt{\frac{1}{3}} \psi_{1 m}\left(\frac{1}{2} \frac{1}{2}\right) .
\end{aligned}
$$

The first eigenvector of Eqs. (38) has the lower eigenvalue since it is determined by choosing the solution to the quadratic eigenvalue equation with the minus sign. Our choice of overall sign for the second eigenvector of Eqs. (38) is opposite that of Eichten and Quigg, but the same as that of Gershtein et al. [7].

\section{RESULTS AND DISCUSSION}

Since the central potential parameters are strictly flavor independent and the constituent masses are not allowed to vary, the only decision that one has to make to address the $B_{c}$ system is to decide on the value of $\alpha_{\bar{S}}$. The simplest choice is the average of the values in the charmonium and the upsilon system, that is, $\alpha_{\bar{S}}=0.393$. This choice requires a value of $\mu=1.12 \mathrm{GeV}$, to preserve the value of $0.190 \mathrm{GeV}$ for the QCD scale. Our results for the low-lying S, P and $\mathrm{D}$ states of the $B_{c}$ system are presented in column 6 of Table $\mathrm{V}$, where they are compared with the predictions of Eichten and Quigg [0], Gershtein et al. [7], Chen and Kuang [16], the NRQCD lattice calculation [35] and our tree level calculation. The energy of each of these states lies below the B-D meson threshold at $7143 \mathrm{MeV}$. In determining the D-state splittings, which are much smaller than the P-state splittings, we followed the example of Eichten and Quigg and did not use the mass-mixing matrix approach. Several running coupling constant effects are noticeable in Table V, although our results are in general agreement with the earlier calculations. Our S-state hyperfine splittings are smaller than those of Eichten and Quigg, and our results for the P-state fine structure splittings are larger. We do not see the inverted order of the D states predicted by both Eichten and Quigg and Gershtein et al.. The order of the fine-structure levels [37,38] is a consequence of the relative sizes of the the perturbative and the nonperturbative contributions to the spin-orbit potentials of Eq. (15).

Comparison with the lattice NRQCD results [35] is of special interest. Their result for the $B_{c}$ mass is based on a lattice calculation of the kinetic mass, $4.76 \pm 0.02$ and a determination of the scale factor by comparison with charmonium and the upsilon system, which gives $a^{-1}=1.32 \pm 0.04 \mathrm{GeV}$. Thus, we list two errors for the lattice calculation of the $B_{c}$ mass in Table $\mathrm{V}$. The smaller error is a consequence of the error in the kinetic mass calculation and the larger error is a consequence of the uncertainty in the overall scale. In determining the other lattice errors, we simply list the largest possible source of error without including the error due to the overall scale. The lattice result for the 1S hyperfine splitting is $41 \pm 3 \mathrm{MeV}$, which is independent of the larger errors discussed above, and somewhat smaller than our $55 \mathrm{MeV}$ result. The lattice result for the $1 P^{2}-1 P^{0}$ splitting is $59 \pm 5 \mathrm{MeV}$, in reasonable agreement with our result of $71 \mathrm{MeV}$. Although the number quoted in Ref. [35] for the $B_{c}$ mass is very close to our result, its importance as a confirmation of our work is undermined by the large error in the overall scale. Another important lattice result is now available from the UKQCD collaboration [36]. Their result for the ground state energy is $6386 \pm 9 \pm 98 \pm 15 \mathrm{MeV}$, which is consistent with our result because of the large error bars. It will be interesting to see whether further refinements of the lattice calculations will support our results, or offer the experimenters alternative predictions.

In order to get some idea of an error estimate for our predictions, we have calculated the ground state mass of the $B_{c}$ system as a function of $\alpha_{\bar{S}}$ in a range bounded by the values determined in charmonium and the upsilon system. Our results are shown in Fig. 1, where they are compared with the result of Table 1, the Eichten-Quigg quote and the NRQCD lattice result. The error shown for the lattice result simply ignores the large error associated with the overall scale. The horizontal lines there describe the limits determined by Kwong and Rosner 断. Using the largest and smallest values of $\alpha_{\bar{S}}$ in Fig. 1 to determine the errors, we have 


$$
M_{B_{c}}=6286_{-6}^{+15} \mathrm{MeV} \quad M_{B_{c}^{*}}=6341_{-5}^{+2} \mathrm{MeV},
$$

as our predicted value for the $1 \mathrm{~S}$ energies. It is interesting to note how close our results are to the earlier predictions of Godfrey and Isgur [14] (6270 and $6340 \mathrm{MeV}$ ) and the predictions of Baker, Ball and Zachariasen [15] (6287 and $6372 \mathrm{MeV}$ ), although our prediction for the hyperfine splitting is smaller than either. Clearly, the precision of the experiments [1] requires a very substantial improvement to be sensitive to the energy differences between the various calculations listed in Table $\mathrm{V}$ and Fig. 1.

The pseudoscalar decay constant is given by the Van Royen-Weisskopf formula modified for color [39], that is,

$$
f_{B_{c}}^{2}=\frac{3\left|R_{1 S}(0)\right|^{2}}{\pi M_{B_{c}}}
$$

and we find that

$$
f_{B_{c}}=517 \mathrm{MeV}
$$

in excellent agreement with Eichten and Quigg's result, $f_{B_{c}}=500 \mathrm{MeV}$, and in reasonable agreement with the lattice result 35, $f_{B_{c}}=440 \pm 20 \mathrm{MeV}$.

The empirical result obtained by Collins et al. 40] for potential model wave functions at the origin, that is,

$$
\left|R_{B_{c}}(0)\right|^{2} \cong\left|R_{J / \psi}(0)\right|^{1.3}\left|R_{\Upsilon}(0)\right|^{0.7},
$$

provides another touchstone for our numerical work. Using this relationship and input from our charmonium and upsilon calculations, we get $\left|R_{B_{c}}(0)\right|^{2} \cong 1.81 \mathrm{GeV}^{3}$, about $3 \%$ higher than our numerical result. Using their empirical relationship for the ground state hyperfine splittings, that is,

$$
M_{B_{c}^{*}}-M_{B_{c}} \simeq 0.7\left(M_{J / \psi}-M_{\eta_{c}}\right)^{0.65}\left(M_{\Upsilon}-M_{\eta_{b}}\right)^{0.35},
$$

yields a splitting of $63 \mathrm{MeV}$, about $14 \%$ larger than our result listed in Table V. Both of these results are reasonable in view of the spread of the results Collins et al. obtained with different forms for the central potential.

Diagonalizing the P-state mixing matrix, we obtain the following combinations for the two lowest $J=1 \mathrm{P}$ states,

$$
\begin{aligned}
& \psi_{1 m}\left(1^{+}\right)=0.118 \psi_{1 m}\left(\frac{3}{2} \frac{1}{2}\right)+0.993 \psi_{1 m}\left(\frac{1}{2} \frac{1}{2}\right), \\
& \psi_{1 m}\left(1^{+^{\prime}}\right)=0.993 \psi_{1 m}\left(\frac{3}{2} \frac{1}{2}\right)-0.118 \psi_{1 m}\left(\frac{1}{2} \frac{1}{2}\right),
\end{aligned}
$$

which is very close to the $\mathrm{j}$-j coupling limit, that one would expect to be valid in the heavy-quark limit. Using the inverse of Eqs. (38), we can determine the probability of observing spin 1 in the lowest $1^{+}$state, $P_{1^{+}}(S=1)=0.773$. Our result is consistent with the lattice calculation [35], where the mixing angle in the L-S basis was found to be close to that of the $\mathrm{j}$-j limit. From this mixing angle $\left(\theta=33.4^{\circ} \pm 1.5^{\circ}\right)$ we obtain $P_{1+}(S=1)=0.697 \pm 0.020$, in reasonable agreement with our result. Our results for the mixing angles of the $1^{+}$and $1^{+^{\prime}}$ states is very different from that of Eichten and Quigg, whose results were much closer to the L-S limit. Below we show that this difference has important implications for the spectrum of photons emitted in electric dipole transitions.

The electric dipole rate for the emission of a photon [5] of energy $\mathrm{k}$ is given by

$$
\Gamma_{E 1}(i \rightarrow f+\gamma)=\frac{4 \alpha\left\langle e_{Q}\right\rangle^{2}}{27} k^{3}\left(2 J_{f}+1\right)|\langle f|r| i\rangle|^{2} S_{i f},
$$

where the statistical factor $S_{i f}=1$ for transitions between triplet $\mathrm{S}$ and triplet $\mathrm{P}$ states [4], and $S_{i f}=3$ for transitions between spin-singlet states. The mean charge in Eq. (45) is

$$
\left\langle e_{Q}\right\rangle=\frac{m_{2} e_{1}-m_{1} e_{2}}{m_{1}+m_{2}},
$$

where $e_{1}$ denotes the charge of the charmed quark (in units of the proton's charge) and $e_{2}$ denotes the charge of the bottom antiquark. Our results for the $1 P \rightarrow 1 S$ and the $2 S \rightarrow 1 P$ transition rates are shown in Table VI, where they are compared with Eichten and Quigg's results. Our rates for transitions involving the $1 P^{2}$ and $1 P^{0}$ states are rather close to their counterparts calculated by Eichten and Quigg. However for the $J=1$ states important differences arise. 
In particular, we predict 4 nonzero transition probabilities instead of 2 . Thus, each of our simulated photon spectra presented in Fig. 2 and Fig. 3 has six lines instead of 4 . These additional lines are a consequence of the fact that our mixture of states is in Eq. (44) is not close to the L-S limit, and thus both $1^{+}$states have a substantial admixture of both triplet and singlet components.

The magnetic dipole transition rate between $\mathrm{S}$ states is given by

$$
\Gamma_{M 1}(i \rightarrow f+\gamma)=\frac{16 \alpha}{3} \mu_{m a q}^{2} k^{3}\left(2 J_{f}+1\right)\left|\left\langle f\left|j_{0}(k r / 2)\right| i\right\rangle\right|^{2},
$$

where the mean magnetic dipole moment is

$$
\mu_{m a g}=\frac{m_{2} e_{1}-m_{1} e_{2}}{4 m_{1} m_{2}} .
$$

Our results for the magnetic dipole transition rates are presented in Table VII, where they are compared with those of Eichten and Quigg [5] and Gershtein et al. [7]. Most of the differences are a consequence of different energies for the hyperfine splittings, since the results for the matrix elements are rather close.

The photon energies and transition rates in Tables VI and VII suggest at least two good strategies for experimental searches for the $1 \mathrm{P}$ and $1 \mathrm{~S}$ states. One could detect one or both of the high energy photons $(457,436 \mathrm{MeV})$ associated with the decay of the lowest two $1^{+}$states to the ground state and then search for a leptonic decay of the ground state. An alternative would be to look for some of the high-energy photons $(417,406,384,350 \mathrm{MeV})$ in the $1 P \rightarrow 1^{3} S_{1}$ transitions and then seek a coincidence with the $55 \mathrm{MeV}$ photon associated with the decay to the ground state.

Since the charmed quark and the bottom antiquark cannot annihilate into gluons, the only additional complication that arises in the decays of these low-lying states is the $\pi \pi$ channel. If we take the rates for the $\pi \pi$ decays from Eichten and Quigg, then we can work out complete decay schemes and branching ratios for the $2 \mathrm{~S}$ and $1 \mathrm{P}$ states as well as the $1^{3} S_{1}$ state. These are shown in Table VIII. Our table of decay rates and branching ratios differs from that of Eichten and Quigg in two important respects. More photon channels are available to the $1^{+}$states and the $2 \mathrm{~S}$ states. Our decay width for the $1^{3} S_{1}$ is $59 \mathrm{eV}$, more than a factor of 2 smaller than theirs.

Our final calculation is that of the lifetime of the ground state of the $B_{c}$ system. We follow the approach used by several researchers 42 44 where the decay of the $B_{c}$ meson is taken to be the sum of 3 distinct contributions, namely weak decay of the $b$ antiquark while the $c$ quark behaves as a spectator, weak decay of the $c$ quark while the $\bar{b}$ antiquark behaves as a spectator and an annihilation of the $\bar{b}$ antiquark and the $c$ quark into an intermediate vector boson that subsequently decays into a lepton-neutrino pair or a quark-antiquark pair. Thus the total decay rate is the sum,

$$
\Gamma\left(B_{c} \rightarrow X\right)=\Gamma(\bar{b} \rightarrow X)+\Gamma(c \rightarrow X)+\Gamma(\text { annih }) .
$$

If one neglects quark binding effects, then the first two terms are given by

$$
\Gamma(\bar{b} \rightarrow X)=\frac{9 G_{F}^{2}\left|V_{c b}\right|^{2} m_{b}^{5}}{192 \pi^{3}}, \Gamma(c \rightarrow X)=\frac{5 G_{F}^{2}\left|V_{c s}\right|^{2} m_{c}^{5}}{192 \pi^{3}},
$$

where the subscripted quantities $V$ denote the appropriate Cabibbo-Kobayashi-Maskawa matrix elements 45] and $G_{F}$ denotes the Fermi coupling constant. Using the constituent masses listed in Eq. (31), we obtain

$$
\Gamma(\bar{b} \rightarrow X)=(8.73 \pm 1.34) \times 10^{-10} \mathrm{MeV}, \Gamma(c \rightarrow X)=(7.59 \pm 0.02) \times 10^{-10} \mathrm{MeV}
$$

The annihilation width is given by

$$
\Gamma(\text { annih })=\frac{G_{F}^{2}}{8 \pi}\left|V_{b c}\right|^{2} f_{B_{c}}^{2} M_{B_{c}} \sum_{i} m_{i}^{2}\left(1-\frac{m_{i}^{2}}{M_{B_{c}}^{2}}\right)^{2} C_{i},
$$

where $m_{i}$ denotes the mass of the heavier Fermion in the given decay channel. The most important channels in the sum are the $\tau \nu$ and the $\bar{c} s$ channels. For the former $C_{i}=1$, and for the latter $C_{i}=3\left|V_{c s}\right|^{2}$. Using Eq. (41) for $f_{B_{c}}$ and Eq. (39) for $M_{B_{c}}$, we have

$$
\Gamma(\text { annih })=(1.13 \pm 0.17) \times 10^{-10} \mathrm{MeV} .
$$

Adding these 3 widths yields a lifetime

$$
\tau_{B_{c}}=0.38 \pm 0.03 p s
$$


in good agreement with the measured CDF result [1]. Our result is also in reasonable agreement with the recent calculation of El-Hady, Lodhi and Vary [14], who obtain $\tau_{B_{c}}=0.46 \mathrm{ps}$, since the spectator widths of Eqs. (50) are very sensitive to small differences in the constituent masses. Although different authors may wish to interprete relatively small differences between $\Gamma(\bar{b} \rightarrow X)$ and $\Gamma(c \rightarrow X)$ as the domination of one process over the other, we feel that the safest characterization of our results is that the two spectator processes are almost equally important and that the annihilation processes are less important, consistent with the earlier conclusion of Gershtein et al. 442].

\section{ACKNOWLEDGEMENTS}

The author gratefully acknowledges the hospitality of Professor Werner Scheid and his colleagues at the Institut für Theoretische Physik of the Justus Liebig Universität. Support for this work was provided by the Alexander von Humboldt-Stiftung.

\section{APPENDIX A: HYPERFINE SPLITTING AND CONTACT POTENTIALS}

Determining the energy shifts from the hyperfine splitting can be problematic because of the delta function that is often present in the spin-spin potential. As Lucha, Schöberl and Gromes point out in their review [24], the energy of the singlet state is not actually bounded from below, in marked contrast to the first-order perturbation theory result of Eq. (3). Although the use of Eq. (3) in the literature is fairly common, seldom does one see any discussion of its validity. It is straightforward to create a context for addressing this question, by considering a more general form for the spin-spin potential, which allows for a finite range, that is,

$$
V_{S S}=\frac{32 \alpha_{S}}{9 m_{1} m_{2}} \frac{e^{-r^{2} / b^{2}}}{\pi^{1 / 2} b^{3}} \mathbf{S}_{1} \cdot \mathbf{S}_{2} .
$$

The advantage of such a form is that one can calculate the singlet and triplet S-state eigenvalues of the Hamiltonian of Eq. (1) exactly and examine the limit as the range parameter $b$ becomes smaller and smaller. We have done such a calculation with the logarithmic potential of Eq. (5) and the parameters listed in Table I. Our results for the exact singlet and triplet as a function of the range $b$ are shown in Fig. 1 , where they are compared with the results of a first-order perturbation calculation of the singlet and triplet energies produced by the potential of Eq. (A1). The contact potential results of Eq. (3) are presented as two horizontal lines. It is gratifying to see the first-order perturbation result from Eq. (A1) above approach the contact potential result in the limit $b \rightarrow 0$. Extrapolating the exact result for the triplet energy to the $b=0$ limit gives $6330 \mathrm{MeV}$, about $3 \mathrm{MeV}$ lower than the result listed in column 4 of Table 1, which was obtained with the contact potential. Thus, it is clear that first-order perturbation theory and the contact potential give a good account of the 1S triplet energy.

Fig. - 1 gives a clear signal of the instability of the singlet energies as $b \rightarrow 0$. However the difficulty begins to appear only as $b$ decreases below $0.3 \mathrm{GeV}^{-1}$. The short range required for the effects of the instability to manifest itself provides a means of resolving this dilemma. As Lucha, Schöberl and Gromes point out, the contact potential expression is not really valid for such short ranges since one must take the nonrelativistic limit in order to obtain it. Such a limit requires that nonlocal effects associated with the normalization of Dirac wave functions be ignored. Thus it might be reasonable to choose $b=0.36 \mathrm{GeV}$, the geometric mean of the Compton wavelengths of the two constituent quarks. Such a choice would lead to a value of the singlet energy very close to that found in column 4 of Table 1.

[1] F. Abe et al. , CDF collaboration, Phys. Rev. D 58112004 (1998).

[2] J. Richardson, Phys. Lett. 82B, 272 (1979).

[3] G. Bali, K. Schilling and A. Wachter, Phys. Rev. D 56, 2566 (1997).

[4] W. Kwong and J. Rosner, Phys. Rev. D 44, 212 (1991).

[5] E. Eichten and C. Quigg, Phys. Rev. D 49, 5845 (1994).

[6] W. Buchmüller and S. Tye, Phys. Rev. D 24, 132 (1981).

[7] S. Gershtein, V. Kiselev, A. Likhoded and A. Tkabladze, Phys. Rev. D 51, 3613 (1995). 
[8] L. Fulcher, Phys. Rev. D 44, 2079 (1991).

[9] S. Gupta and S. Radford, Phys. Rev. D 24, 2309 (1981); 25, 2690 (1982).

[10] S. Gupta, S. Radford and W. Repko, Phys. Rev. D 26, 3305 (1982); 34, 201 (1986).

[11] J. Pantaleone, S. Tye and Y. Ng, Phys. Rev. D 33, 777 (1986).

[12] D. Gromes, Zeit. Phys. C 26 , 401 (1984).

[13] E. Eichten and F. Feinberg, Phys. Rev. D 23, 2724 (1981).

[14] S. Godfrey and N. Isgur, Phys. Rev. D 32, 189 (1985).

[15] M. Baker, J. Ball and F. Zachariasen, Phys. Rev. D 45, 910 (1992).

[16] Y. Chen and Y. Kuang, Phys. Rev. D 46, 1165 (1992).

[17] V. Galkin, A. Mishurov and R. Faustov, Sov. J. Nucl. Phys. 55, 1207 (1992).

[18] L. Fulcher, Phys. Rev. D 37, 1258 (1988).

[19] E. Eichten et al., Phys. Rev. D 17, 3090 (1978); 21, 203 (1980).

[20] A. Martín, Phys. Lett. 93B, 338 (1980).

[21] C. Quigg and J. Rosner, Phys. Lett. 71B, 153 (1977).

[22] X. Song and H. Lin, Zeit. Phys. C 34, 223 (1987).

[23] D. Lichtenberg et al., Zeit. Phys. C 41, 615 (1989).

[24] W. Lucha, F. Schöberl and D. Gromes, Phys. Rep. 200, 127 (1991).

[25] D. Griffiths, Introduction to Elementary Particles (Harper and Row, New York, 1987).

[26] In the present work we choose $n_{f}=3$ to prevent introducing further parameters.

[27] P. Moxhay and J. Rosner, Phys. Rev. D 28, 1132 (1983).

[28] L. Landau and E. Lifshitz, Quantum Mechanics (Addison-Wesley, Reading, MA, 1965).

[29] W. Kwong and J. Rosner, Phys. Rev. D 38, 279 (1988).

[30] W. Lucha and F. Schöberl, Die Starke Wechselwirkung (BI Wissenschaftsverlag, Mannheim, 1989).

[31] Particle Data Group, R. Barnett et al. Phys. Rev. D 54, 1 (1996).

[32] N. Brambilla and A. Vairo, Phys. Rev. D 55, 3974 (1997).

[33] F. Close, An Introduction to Quarks and Partons (Academic, New York, 1979).

[34] W. Kwong, P. Mackenzie, R. Rosenfeld and J. Rosner, Phys. Rev. D 37, 3210 (1988).

[35] C. Davies, K. Hornbostel, G. Lepage, A. Lidsey, J. Shigemitsu and J. Sloan, Phys. Lett. B 382 , 131 (1996).

[36] H. Shanahan, P. Boyle, C. Davies and H. Newton, UKQCD collaboration, available as lanl eprint hep-lat/9902025.

[37] H. Schnitzer, Nucl. Phys. B207, 131 (1982).

[38] N. Isgur, Phys. Rev. D 57, 4041 (1998).

[39] R. Van Royen and V. Weisskopf, Nuovo Cimento 50 , 617 (1967); 51, 583 (1967).

[40] S. Collins, T. Imbo, B. King and E. Martell, Phys. Lett. B 393, 155 (1997).

[41] E. Eichten and K. Gottfried, Phys. Lett. 66B, 286 (1977).

[42] S. Gershtein, A. Likhoded and S. Slabospitsky, Int. Jour. Mod. Phys. A 6, 2309 (1991).

[43] I. I. Bigi, Phys. Lett. B 371, 105 (1996).

[44] A. El-Hady, M. Lodhi and J. Vary, Phys. Rev. D 59, 094001 (1999).

[45] Particle Physics Booklet, C. Caso et al., July 1998.

FIG. 1. Ground state energy of the $B_{c}$ system as a function of the running coupling constant. The PH98 result is based on the calculations presented in Table $\mathbb{\Psi}$.

FIG. 2. Simulated photon spectrum for $1 P \rightarrow 1 S$ transitions. The probability of populating each of the initial states is assumed to be equal.

FIG. 3. Simulated photon spectrum for $2 S \rightarrow 1 P$ transitions.

FIG. 4. Hyperfine splittings of the $1 \mathrm{~S}$ state for a finite range spin-spin potential. 
TABLE I. Ground state energies of the heavy-quark systems (MeV).

\begin{tabular}{|c|c|c|c|c|c|}
\hline$\overline{\text { State }}$ & Cornell & Martín & logarithm & Song-Lin & Turin \\
\hline$\overline{\alpha_{S}}$ & 0.313 & 0.437 & 0.372 & 0.396 & 0.373 \\
\hline$m_{b}(G e V)$ & 5.232 & 5.174 & 4.905 & 5.199 & 5.171 \\
\hline$m_{c}(G e V)$ & 1.840 & 1.800 & 1.500 & 1.820 & 1.790 \\
\hline $1^{1} S_{0}$ & 2980 & 2980 & 2980 & 2980 & 2980 \\
\hline$\Delta E_{1 S}$ & 117 & 117 & 117 & 117 & 117 \\
\hline $1^{3} S_{1}(b \bar{b})$ & 9461 & 9461 & 9460 & 9460 & 9460 \\
\hline$\Delta E_{1 S}$ & 145 & 64 & 65 & 80 & 95 \\
\hline $1^{3} S_{1}(c \bar{b})$ & 6337 & 6319 & 6333 & 6324 & 6327 \\
\hline $1^{1} S_{0}$ & 6246 & 6247 & 6266 & 6247 & 6247 \\
\hline$\Delta E_{1 S}$ & 91 & 72 & 67 & 77 & 80 \\
\hline
\end{tabular}

TABLE II. Charmonium energies (MeV).

\begin{tabular}{lcccc}
\hline \hline State & EQ94 & FUI98 & FUII98 & EXPT \\
\hline $1^{3} S_{1}$ & 3097 & 3098 & 3098 & $3097 \pm 0.04$ \\
$1^{1} S_{0}$ & 2980 & 2981 & 2980 & $2980 \pm 2$ \\
$2^{3} S_{1}$ & 3686 & 3692 & 3693 & $3686 \pm 0.1$ \\
$2^{1} S_{0}$ & 3608 & 3617 & 3615 & $355 \pm \pm 0.1$ \\
$1^{3} P_{2}$ & 3507 & 3515 & 3530 & $3511 \pm 0.1$ \\
$1^{3} P_{1}$ & 3486 & 3492 & 3482 & $3415 \pm 1.0$ \\
$1^{3} P_{0}$ & 3436 & 3443 & 3391 & $3526 \pm 0.1$ \\
$1^{1} P_{1}$ & 3493 & 3499 & 3501 & 19.9 \\
$\sqrt{\delta^{2}}$ & 25.5 & 22.7 & 350 \\
\hline \hline
\end{tabular}

TABLE III. Upsilon energies (MeV).

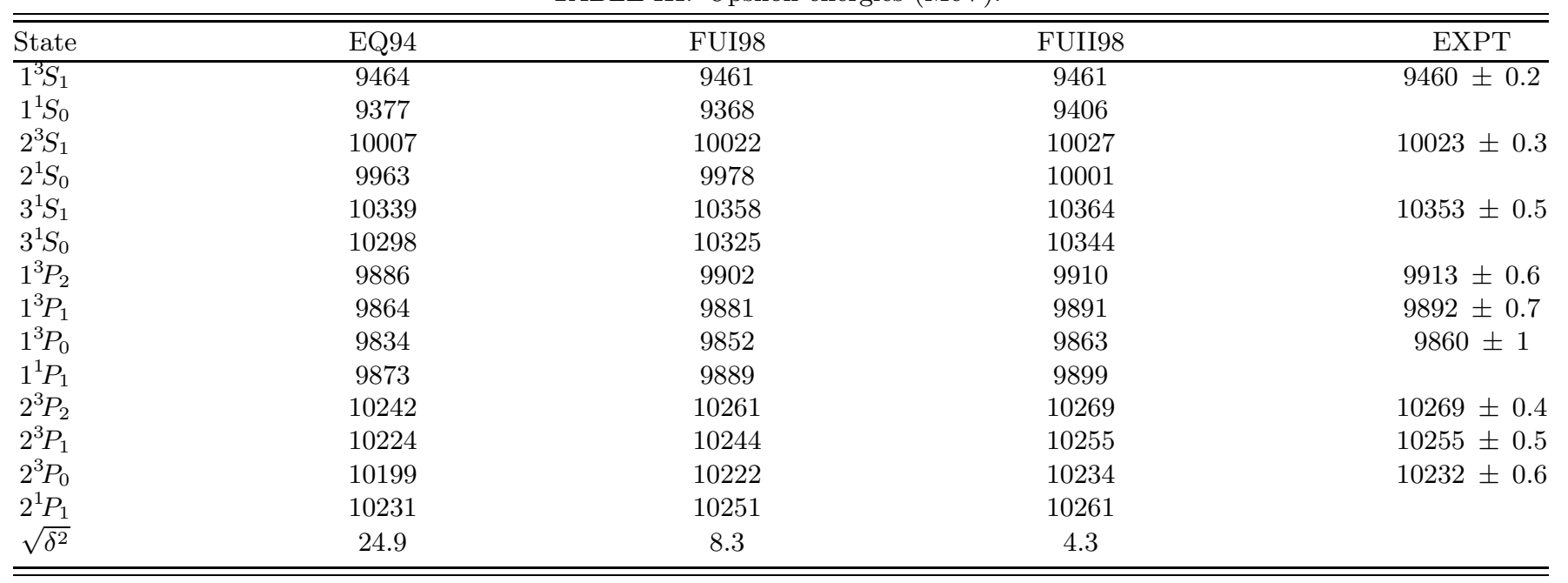


TABLE IV. Leptonic widths $(\mathrm{KeV})$.

\begin{tabular}{lccc}
\hline \hline State & EQ94 & FU98 & EXPT \\
\hline$\Upsilon(1 S)$ & $1.71(1.19)$ & 1.34 & $1.32 \pm 0.05$ \\
$\Upsilon(2 S)$ & $0.76(0.53)$ & 0.57 & $0.52 \pm 0.03$ \\
$\Upsilon(3 S)$ & $0.55(0.38)$ & 0.40 & $0.48 \pm 0.06$ \\
$\psi(1 S)$ & $8.00(5.55)$ & 5.81 & $5.26 \pm 0.37$ \\
$\psi(2 S)$ & $3.67(2.55)$ & 2.61 & $2.14 \pm 0.21$ \\
\hline \hline
\end{tabular}

TABLE V. Energies of the $B_{c}$ system $(\mathrm{MeV})$.

\begin{tabular}{|c|c|c|c|c|c|c|}
\hline$\overline{\text { State }}$ & EQ94 & GKLT95 & CK92 & FUI98 & FUII98 & LAT96 \\
\hline$\overline{1^{3} S_{1}}$ & 6337 & 6317 & 6355 & 6341 & 6341 & $6321 \pm 30$ \\
\hline $1^{1} S_{0}$ & 6264 & 6253 & 6310 & 6267 & 6286 & $6280 \pm 30 \pm 190$ \\
\hline $2^{3} S_{1}$ & 6899 & 6902 & 6917 & 6911 & 6914 & $6990 \pm 80$ \\
\hline $1 P_{2}$ & 6747 & 6743 & 6773 & 6761 & 6772 & $6783 \pm 30$ \\
\hline $1 P 1^{+^{\prime}}$ & 6736 & 6729 & & 6750 & 6760 & $6765 \pm 30$ \\
\hline $1^{3} D_{3}$ & 7005 & 7007 & & 7022 & 7032 & \\
\hline $1^{3} D_{2}$ & 7012 & & & 7025 & 7028 & \\
\hline $1^{3} D_{1}$ & 7012 & & & 7024 & 7019 & \\
\hline $1^{1} D_{2}$ & 7009 & 7008 & & 7023 & 7028 & \\
\hline
\end{tabular}

TABLE VI. Electric dipole matrix elements and transition rates.

\begin{tabular}{|c|c|c|c|c|c|c|}
\hline \multirow[t]{2}{*}{ Transition } & \multirow{2}{*}{$\begin{array}{c}\text { Photon energy } \\
(\mathrm{MeV})\end{array}$} & \multirow[b]{2}{*}{ GKLT95 } & \multirow{2}{*}{$\begin{array}{c}\langle f|r| i\rangle(\mathrm{GeV})^{-1} \\
\text { EQ94 }\end{array}$} & \multicolumn{3}{|c|}{$\Gamma(K e V)$} \\
\hline & & & & FU98 & EQ94 & FU98 \\
\hline $1 P_{2} \rightarrow 1^{3} S_{1}$ & 417 & & & & 113 & 126 \\
\hline $1 P 1^{+^{\prime}} \rightarrow 1^{3} S_{1}$ & 406 & & & & 0.1 & 26.2 \\
\hline $1 P 1^{+} \rightarrow 1^{3} S_{1}$ & 384 & & & & 99.5 & 75.8 \\
\hline $1 P_{0} \rightarrow 1^{3} S_{1}$ & 350 & 1.568 & 1.714 & 1.683 & 79.2 & 74.2 \\
\hline $1 P 1^{+^{\prime}} \rightarrow 1^{1} S_{0}$ & 457 & & & & 56.4 & 128 \\
\hline $2^{3} S_{1} \rightarrow 1 P_{2}$ & 141 & & & & 17.7 & 14.5 \\
\hline $2^{3} S_{1} \rightarrow 1 P 1^{+^{\prime}}$ & 152 & & & & 0.0 & 2.5 \\
\hline $2^{3} S_{1} \rightarrow 1 P 1^{+}$ & 175 & & & & 14.5 & 13.3 \\
\hline $2^{3} S_{1} \rightarrow 1 P_{0}$ & 210 & -2.019 & -2.247 & -2.253 & 7.8 & 9.6 \\
\hline $2^{1} S_{0} \rightarrow 1 P 1^{+^{\prime}}$ & 121 & & & & 5.2 & 13.1 \\
\hline
\end{tabular}

TABLE VII. Magnetic dipole matrix elements and transition rates.

\begin{tabular}{lcccrr}
\hline \hline Transition & $\begin{array}{c}\text { Photon energy } \\
(\mathrm{MeV})\end{array}$ & $\left\langle f\left|j_{0}(k r / 2)\right| i\right\rangle$ & & \multicolumn{2}{c}{$\Gamma(\mathrm{KeV})$} \\
& EQ94 & FU98 & GKLT95 & EQ94 & FU98 \\
\hline $2^{3} S_{1} \rightarrow 2{ }^{1} S_{0}$ & 52 & 0.9990 & 0.9995 & 0.010 & 0.029 \\
$2{ }^{3} S_{1} \rightarrow 1{ }^{1} S_{0}$ & 599 & 0.0395 & 0.0399 & 0.098 & 0.123 \\
$2{ }^{1} S_{0} \rightarrow 1{ }^{3} S_{1}$ & 55 & 0.0265 & 0.0305 & 0.096 & 0.122 \\
$1^{3} S_{1} \rightarrow 1{ }^{1} S_{0}$ & 9.9993 & 0.9996 & 0.060 & 0.093 & 0.135 \\
\hline \hline
\end{tabular}


TABLE VIII. Decays and branching ratios of the $B_{c}$ system.

\begin{tabular}{|c|c|c|c|}
\hline State & Total width $(\mathrm{KeV})$ & Decay mode & Branching ratio (percent) \\
\hline$\overline{1^{3} S_{1}}$ & 0.059 & $1^{1} S_{0}+\gamma$ & 100 \\
\hline $1 P_{2}$ & 126 & $1^{3} S_{1}+\gamma$ & 100 \\
\hline $1 P 1^{+^{\prime}}$ & 154 & $\begin{array}{l}1{ }^{3} S_{1}+\gamma \\
1^{1} S_{0}+\gamma\end{array}$ & $\begin{array}{l}17 \\
83\end{array}$ \\
\hline $1 P 1^{+}$ & 108 & $\begin{array}{l}1^{3} S_{1}+\gamma \\
1^{1} S_{0}+\gamma\end{array}$ & $\begin{array}{l}70 \\
30\end{array}$ \\
\hline $1 P_{0}$ & 74.2 & $1^{3} S_{1}+\gamma$ & 100 \\
\hline $2^{3} S_{1}$ & $89.9 \pm 7$ & $\begin{array}{l}1^{3} S_{1}+\pi \pi \\
1 P_{2}+\gamma \\
1 P 1^{+^{\prime}}+\gamma \\
1 P 1^{+}+\gamma \\
1 P_{0}+\gamma\end{array}$ & $\begin{array}{l}56 \pm 8 \\
16 \pm 1 \\
3 \pm 0.2 \\
15 \pm 1 \\
11 \pm 1\end{array}$ \\
\hline $2^{1} S_{0}$ & $69.5 \pm 7$ & $\begin{array}{l}1^{1} S_{0}+\pi \pi \\
1 P 1^{+^{\prime}}+\gamma \\
1 P 1^{+}+\gamma\end{array}$ & $\begin{array}{c}72 \pm 10 \\
19 \pm 2 \\
9 \pm 1\end{array}$ \\
\hline
\end{tabular}




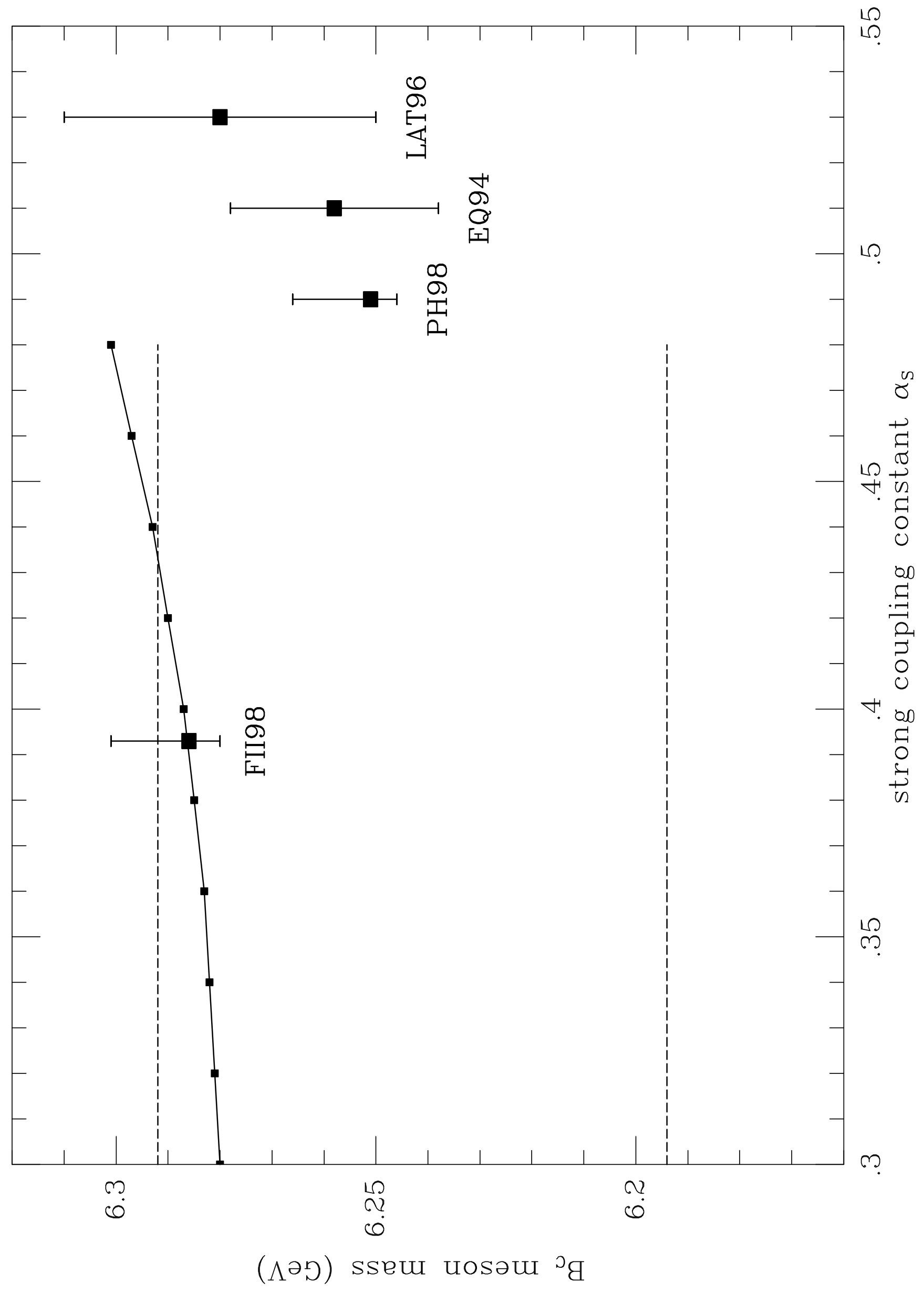




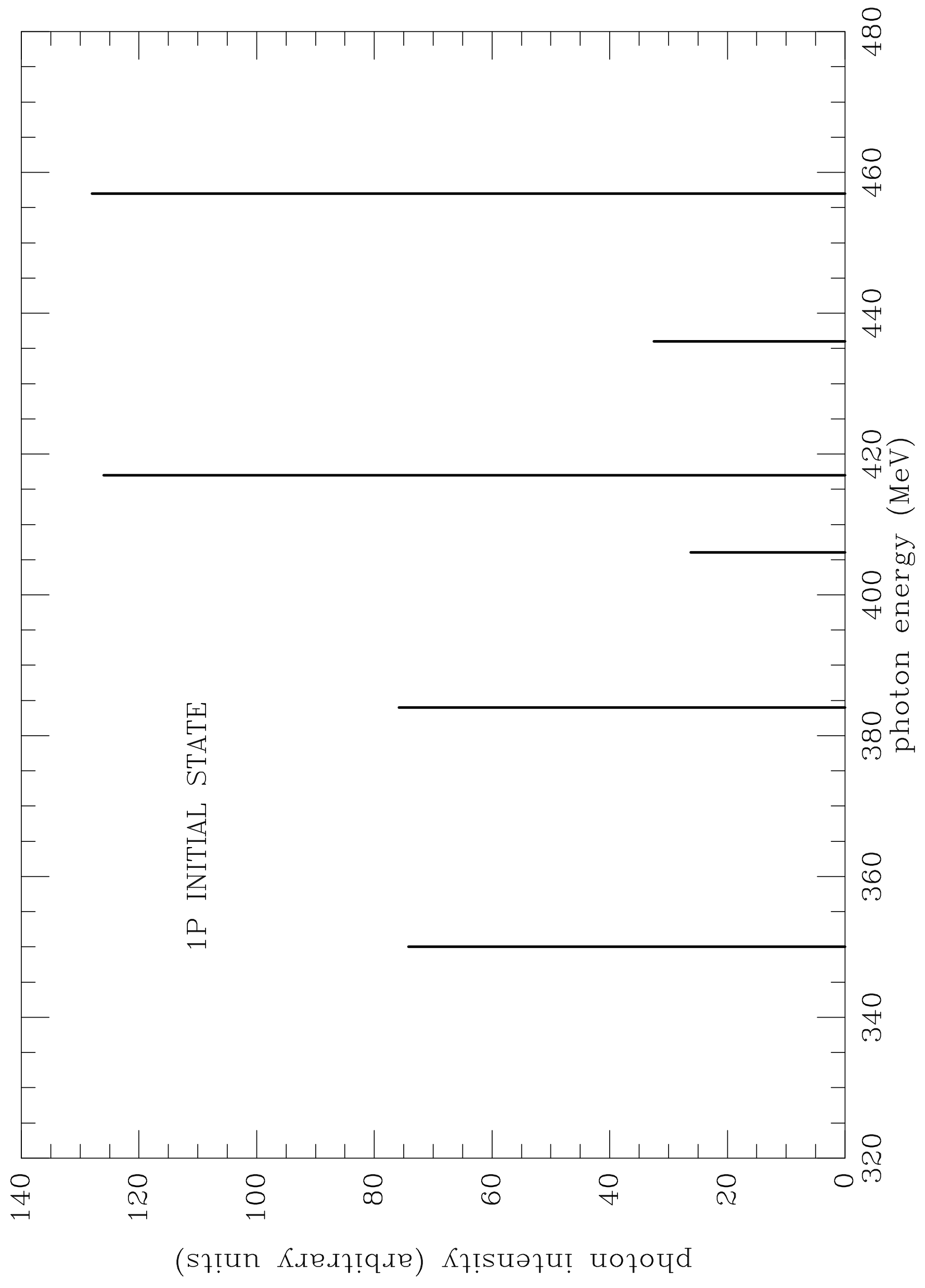




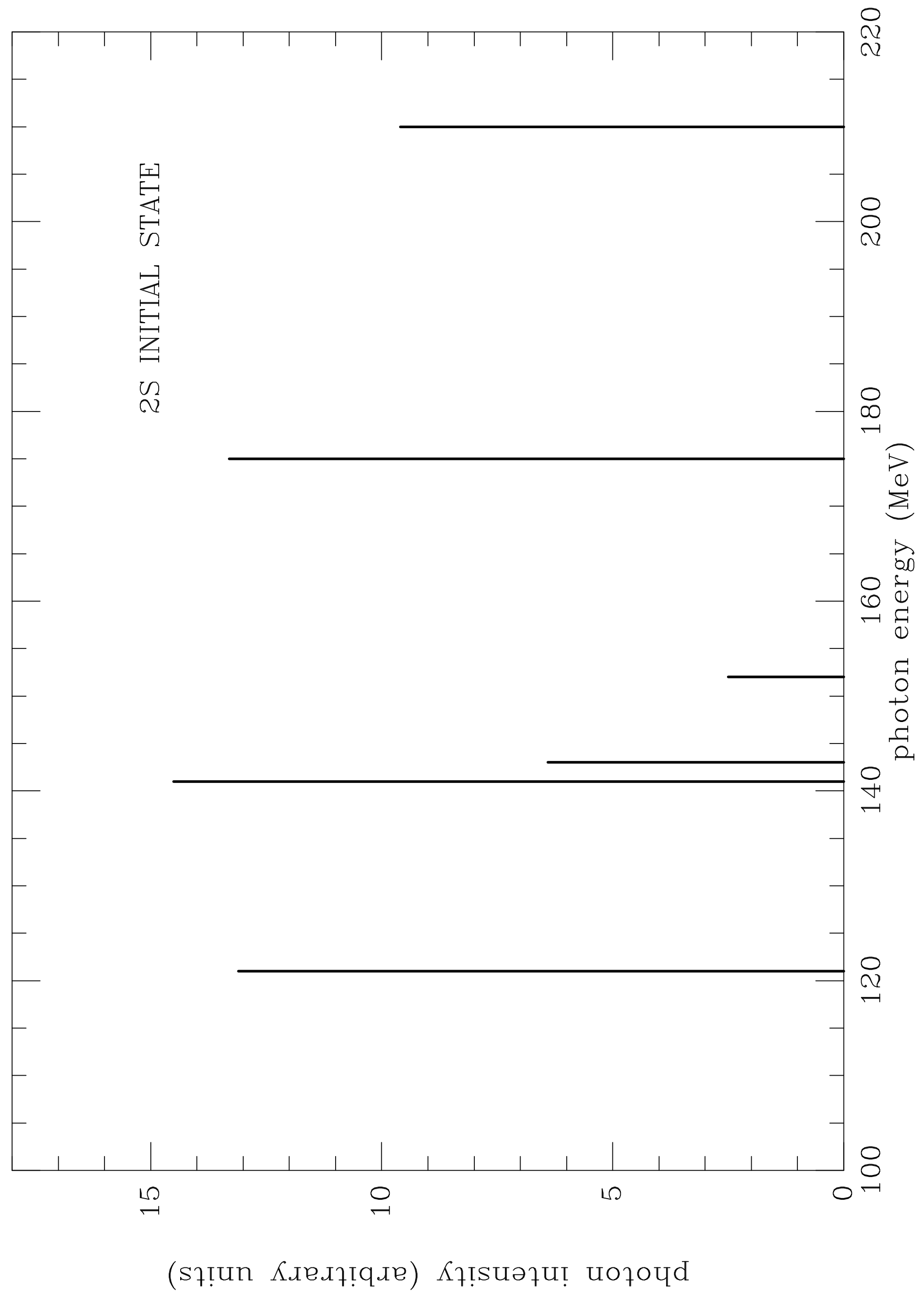




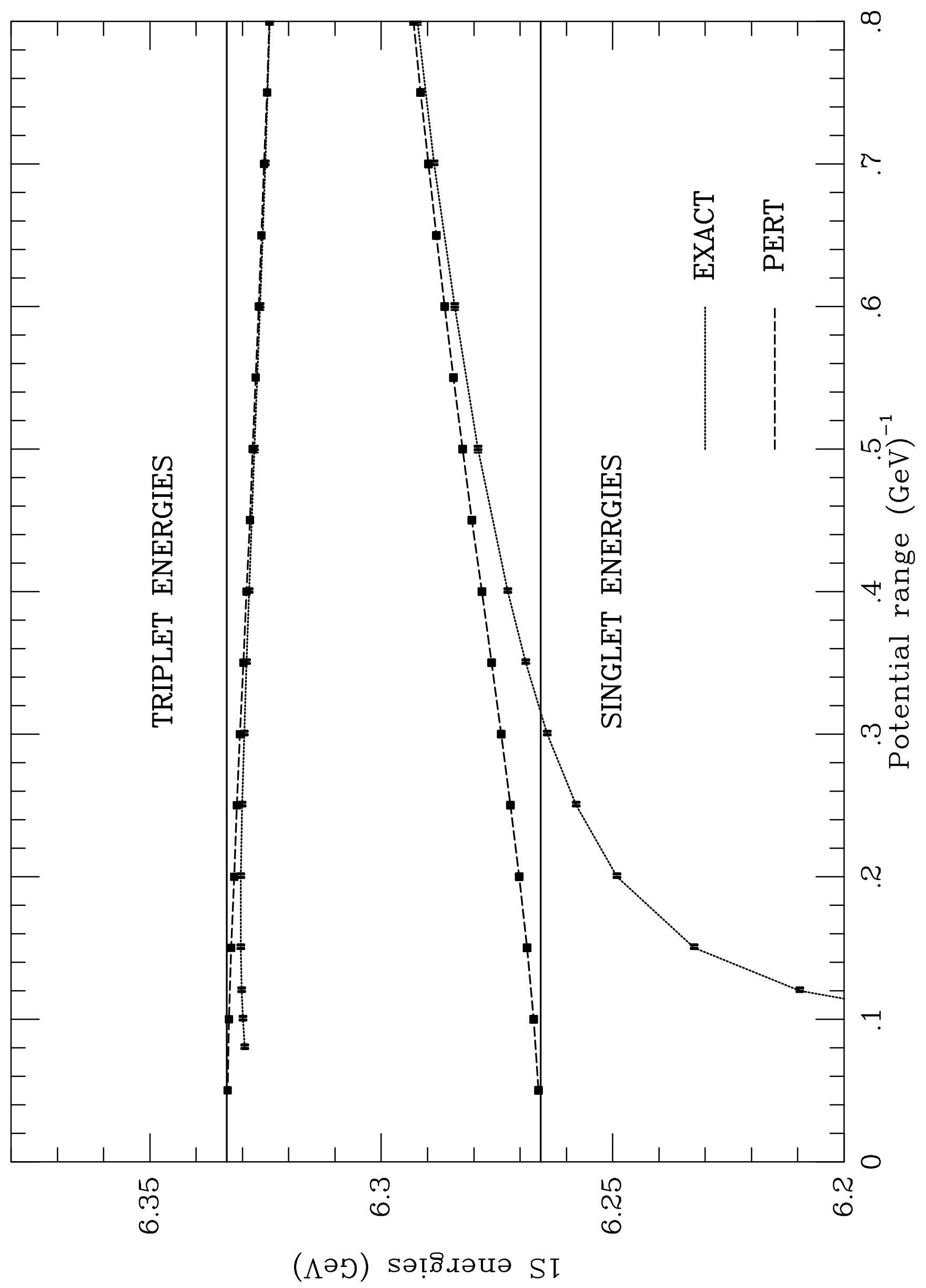

